\title{
Carcinome verruqueux oral traité par perfusions de méthotrexate : à propos d'un cas
}

\section{D’Elbée JM, Jouary T, Boralevi F, Fricain JC (UFR Odontologie Université Segalen, Pôle d’Odontologie et de Santé buccale, Bordeaux)}

Le carcinome verruqueux oral (CV0) a été décrit pour la première fois en 1948 par Ackerman qui le considérait comme un variant non invasif du carcinome épidermoïde oral (Ackerman et al, 1948). Les facteurs étiologiques et la pathogenèse du CV0 font encore débat. Le tabac fumé et chiqué, l'alcool seraient des facteurs de risque important (Walvekar et al. 2009). Des études récentes écartent le HPV comme facteur de risque de CVO (Stokes et al. 2012). Bien qu'il ne métastase pas, la morbidité peut être importante du fait des récurrences locales fréquentes et du risque d'évolution en carcinome épidermoïde (Walvekar et al. 2009). L'exérèse chirurgicale est considérée comme le traitement de choix (Huang et al. 2009). La radiothérapie et plus récemment la chimiothérapie par méthotrexate ont été décrites comme des techniques efficaces dans les formes étendues (Karagozoglu et al, 2012). Un cas de CVO étendu traité avec succès par méthotrexate est rapporté.

Une femme de 69 ans était adressée pour avis concernant une importante kératose intra orale et labiale, résistante aux traitements locaux. Ses antécédents étaient un carcinome épidermoïde gingivo palatin traité par chirurgie et radiothérapie, une hypertension artérielle, un asthme, un reflux gastro oesophagien et un tabagisme évalué à 10 PA sevré depuis 20 ans. L'examen clinique révélait une importante leuco kératose du palais et de la face interne de la joue droite s'étendant sur la lèvre inférieure et la commissure labiale droite compatible avec le diagnostic de carcinome verruqueux. Les aires ganglionnaires étaient libres. L'aspect histologique était en faveur d'un carcinome verruqueux avec un épithélium parakératosique, bien différencié, hyper papillomateux et mamelonné, des crêtes épithéliales larges repoussant sans l'envahir le chorion sous jacent qui présentait un infiltrat inflammatoire. Aucune atypie cellulaire n'était retrouvée. Un traitement par deux cures de methotrexate à la dose de $40 \mathrm{mg} / \mathrm{m}^{2}$ (soit $67 \mathrm{mg}$ ) en intra veineux à 8 jours d'intervalle fut débuté. Après la première cure, on notait une régression nette des lésions. La deuxième cure fut associée à des bains de bouche de solupred pour limiter l'inflammation locale, la gène à l'alimentation et la mucite. Du miconazole fut également prescrit pour traiter une surinfection fungique. Compte tenu de la persistance de lésions résiduelles et de la très bonne tolérance clinique et biologique des deux premières cures, deux nouvelles cures d'entretien furent programmées un mois plus tard à 15 jours d'intervalle. Elles étaient associées à $60 \mathrm{mg}$ de Solupred ${ }^{\circledR}$ per os pendant les 3 jours suivant l'injection, des bains de bouche de Solupred ${ }^{\circledR}$ et des antifongiques. Les effets secondaires étaient essentiellement muqueux (mucite) et furent bien tolérés. La rémission était totale.

Ce résultat encourageant suggère que les perfusions de methotrexate pourraient être une alternative favorable à la radiothérapie et à la chirurgie dans le traitement des formes diffuses de CVO. Le choix de la voie d'administration, de la dose et de la fréquence des cures a été fait en fonction des résultats publiés par Van der Wall (2011) sur une série de 12 cas. L'efficacité et les posologies optimales d'utilisation des perfusions de methotrexate dans le traitement du CVO demandent cependant à être précisées dans des études cliniques. 